\title{
Education, Training, and Mentorship of Caregivers of Canadians Experiencing a Life-Limiting Illness
}

\author{
Allison M. Williams, PhD
}

\begin{abstract}
Background: Research suggests that caregiver preparedness is essential to minimizing the negative impacts of caregiving. Not being prepared is associated with fear, anxiety, stress, and feelings of insufficiency/uncertainty specific to the caregiver role.

Objective: To determine what resources are required to ensure adequate education, training, and mentorship for caregivers of Canadians experiencing a life-limiting illness.

Design: Informed by the Ispos Reid survey, the methods for this article involved a rapid literature review that addressed caregiver experiences, needs and issues as they related to health, quality of life, and well-being.

Results: Given the burden of care, caregiver education, training, and mentorship are suggested to be best met through the palliative navigator model, wherein the patient-caregiver dyad is recognized as an integrated unit of care.

Conclusions: The palliative navigator approach is a key role in the education, training, and mentorship of caregivers.
\end{abstract}

Keywords: navigator; palliative caregivers; patient-caregiver dyad

\section{Introduction}

$\mathrm{C}$ AREgivers of Canadians experiencing a life-limiting illness have been recognized as the backbone of our healthcare system, saving millions of healthcare dollars. Although many caregivers are happy to care and receive many rewards from providing care, others feel that they have very little, if any, voice in taking on the caregiving role.

Research suggests that caregiver preparedness is essential to minimizing the negative impacts of caregiving. Not being prepared is associated with fear, anxiety, stress, and feelings of insufficiency and uncertainty specific to the caregiver role. ${ }^{1-6}$

Given this, Canadian society has to ensure that palliative and end-of-life (EOL) caregivers receive the support they need through adequate caregiving education, training, mentorship, and support services. These supports need to be tailored to the caregiver's circumstances to adequately ensure they can provide care throughout the required period of time and do so feeling confident and effective.

As informed by the Ispos Reid survey introduced earlier in this collection, this article sets out to determine the resources required to ensure adequate education, training, and mentorship for caregivers of Canadians experiencing a life- limiting illness. As outlined, the methods for this article involved a rapid literature review that addressed caregiver experiences, needs and issues as they related to health, quality of life, and well-being. In combination, these methods produced the results and findings specific to education, training, and mentorship. The article begins with a short contextual introduction to the issue, followed by the presentation of the results and implications thereof, which outline the resources required for a suitable education, training, and mentorship of caregivers of Canadians experiencing a life-limiting illness. Finally, conclusions are stated.

\section{Ipsos report findings}

Polled Canadians feel that confidence in palliative/EOL caregiving is moderate and Canadians prefer to be trained by those in the health system by hospital healthcare providers, such as physicians and nurses. ${ }^{7}$ The following are findings from the report provided by Ipsos from a survey conducted during the summer of 2016 for the Palliative Care Matters initiative:

- Almost one-quarter (24\%) of Canadians have been $(20 \%)$ or are currently $(4 \%)$ caregiving for someone 
important to them who was or is dealing with a lifethreatening illness, and among this subgroup, $77 \%$ are confident in providing the necessary care.

- If Canadians were caring for a close friend or family member who was dealing with a life-threatening illness, priority information needs involve who to call in an emergency (73\%), how to use machines $(72 \%)$, what to do when a patient dies at home (72\%), how to give medication $(71 \%)$, and information about dedicated care providers $(70 \%)$. Canadians would also seek information about options for financial support (68\%), the patient's diet $(67 \%)$, and what supplies are needed (64\%), among other topics.

- Canadians feel that hospitals or healthcare facilities in which the palliative care is being provided (46\%) should be responsible for providing information and training for caregivers. Just more than 3-in-10 Canadians place such responsibility on family doctors (32\%) and nurses $(31 \%)$, followed by provincial $(29 \%)$ and federal (21\%) governments.

- A strong majority of Canadians agree that all 15 elements of a potential palliative home care program should be included. The strongest agreement is found for having 24/7 access to a nurse (91\%), daily assistance with caregiving (91\%), access to equipment as needed $(91 \%)$, panic alerts/buttons for patients when left alone $(91 \%)$, education and information for caregivers $(91 \%)$, someone in the system to coordinate care $(91 \%)$, and a single access point (i.e., one number to call) for help (90\%). The lowest agreement expressed (although still strong at $80 \%$ ) is found for renovation funding (i.e., bathroom bars, ramps, and vehicles).

Furthermore, there are a wide range of education, training, and mentorship resources offered by a range of stakeholders, which complement the "on the job" training provided by palliative care and primary care resources that may be available to palliative/EOL caregivers. These include the Canadian Virtual Hospice, which offers various on-line resources, from videos to webcasts; the Canadian Hospice Palliative Care Association, which offers a range of resources on Family Caregiving for People at the End of Life; the Family Caregivers Network Society offers support to those living in British Columbia, although their on-line resources are accessible to all; the Care to Know Centre, which moderates discussion forums on a range of caregiver and diseasespecific topics; Glaxo Smith Kline offers its Living Lessons Campaign; and others such as the Alzheimer Society of Canada, The Victorian Order of Nurses, and Carers Canada.

\section{Results and Findings}

The results of the rapid literature review, together with the knowledge gleaned from a 20 -year research career addressing caregiver experiences, suggest three interlinking themes: caregiver burden, caregiver education, and the role of the palliative navigator.

\section{The burden of care}

Statistic Canada's 2007 General Social Survey Cycle 21 suggests that the majority of palliative/EOL caregivers are female, married, employed, and living in a Census Metropolitan Area, which is defined as a population of at least 100,000 with half of those people living in the core area. ${ }^{5}$

When compared with short- or long-term caregivers of people with a chronic condition or long-term illness (less than two years or more than two years), palliative/EOL caregivers were found to assess their own health status as significantly lower; specifically, they rated themselves more often as having "fair or poor" levels of the health. They were also more likely to be negatively impacted by the often additional role of caregiving. They experienced a higher proportion of negative impacts on their social and activity patterns and incurred greater financial costs. Being a palliative/EOL caregiver also had a negative impact on their ability to work.

This suggests that palliative/EOL caregiving is the most intense type of caregiving, potentially causing the greatest caregiver burden. ${ }^{5}$ Palliative/EOL caregivers also experience complex transitions in their roles and relationships, environment and physical, and mental health while they are providing palliative/EOL care. This has been found to decrease their quality of life. ${ }^{8}$

\section{Educating caregivers}

One of the primary challenges to educating family caregivers is that they often do not see themselves as legitimate recipients of supportive services. Assessing their requirements for support, while important, needs to be done in the context of also focusing on what the person they are caring for requires. 9,10

Although this is the case, Canadians want education, training, and mentorship to be a person-to-person exercise wherein they are supported or guided, either by being shown or told, by others who are trained in palliative care. There are a number of strategies that support the learning process. Caregivers learn through trial and error, actively seeking needed information and guidance, applying experienced knowledge and skills, and reflecting on their current experiences. ${ }^{1-13}$ Supported learning processes are usually initiated after a crisis and involve being shown or told by others. ${ }^{11-13}$

\section{Role of a palliative navigator}

The Ipsos finding that responsibility for education lies with family doctors or nurses suggests a medicalized and expensive approach. ${ }^{5}$ Similar to the midwifery model, the idea of a palliative navigator has been suggested by a number of scholars and practitioners. ${ }^{14,15}$ The role of the navigator would be to work side by side with families, providing a single access point and assisting in care coordination.

Caregivers prefer to have person-to-person learning that is complemented by secondary or tertiary resources, such as those available on-line, through telephone, or in hard copy formats. This provides the opportunity for the palliative navigator to give effective, individualized training and to be able to address the demographic variations and affiliations of any individual caregiver. ${ }^{16}$ These include differences resulting from geography (i.e., urban vs. rural), ${ }^{17}$ culture (i.e., settler vs. First Nation or New Canadian), ${ }^{18}$ employment status (i.e., self-employed, fulltime, and part-time), sex and gender, religion (i.e., Christian vs. Islam), and socioeconomic status (i.e., impoverished vs. well-off), among others. ${ }^{19}$ 


\section{Discussion/Implications}

Recognizing the importance of the patient-caregiver dyad as an integrated unit of care is essential to the role of the palliative navigator, which essentially educates, trains, and mentors the family caregiver while keeping a pulse on the patient's situation.

\section{The patient-caregiver dyad}

The findings suggest that healthcare providers and palliative navigators, irrespective of the setting of care (i.e., hospital, long-term care, or home/community), recognize that the unit of care is not only the patient but also the patientcaregiver dyad. Just as the patient needs care, the caregiver needs training and mentorship in providing quality, tailored care, which will provide confidence and effectiveness in the caregiving role. They also need support specifically to help them deal with the transitions they experience as a caregiver. The care of the dyad needs to happen simultaneously, where the caregiver is being trained and mentored as the patient is being cared for. Supporting caregivers is essential to the care of the palliative dyad unit and is reflected in the quality of care provided to the individual who is dying.

When family caregivers and healthcare providers establish good working relationships, quality of care is enhanced, care delivery is more efficient, and requests by family caregivers for inappropriate levels of service are reduced. For caregivers, that relationship adds to a sense of security that improves their ability to cope with their situation and helps them better understand their role in the delivery of care.

\section{The palliative navigator}

A number of possible approaches to meeting these needs have been suggested, including many individual and group educational and training interventions using different mediums for delivery. Caregivers have a great range of need and significant variations in their profiles, including gender, sex, employment status, culture, and geography. Therefore, caregiver needs would ideally be best met through a palliative navigator, particularly in smaller rural communities wherein there may be closer community connections and cohesion.

Similar to a midwife for a pregnant woman who assists in realizing the safe delivery of new life into the world, a palliative navigator would ensure that the caregiver-patient dyad have their needs best met at EOL. Such a navigator would be available for ongoing consultation around a caregiver's educational, training, and mentoring needs. Similar to the midwifery model, where a family is given a binder of educational and training resources at the beginning of the midwife-family relationship, a palliative navigator would do the same, putting in place a foundation of knowledge as a "go to" tool for caregivers. Similarly, regular check-ins and meetings would nurture a trusting relationship. This relationship would become more intense at the time of death. After the death of the patient, bereavement issues would be addressed with the assistance of the palliative navigator. Taken one step further, the palliative navigator would also be a member of, where available, the palliative care team, which is an interdisciplinary team of practitioners (including social work and spiritual care) that have shown to be particularly effective in rural areas. ${ }^{7,20}$

\section{Education, training, and mentorship}

The provision of education, training, and mentorship requires much of the same support and strategies, although they each represents different aspects of the information and support required by caregivers.

Education encompasses how to access help such as healthcare, community supports, transportation, caregiverfriendly programs in the workplace, living wills, and advanced care planning ${ }^{21}$ in addition to general information about the patient's disease(s) and what to expect. ${ }^{1,22}$ Information preferences vary and often depend upon the ability to comprehend and/or accept the terminal diagnosis. ${ }^{23}$ Furthermore, disease-specific education needs to take place throughout the illness trajectory. ${ }^{24-26}$ Two additional educational needs identified are financial planning/budgeting and effective communication, especially as it relates to information sharing needed between healthcare providers, caregiver, and patient. ${ }^{27}$

Training includes the practical aspects of care, including personal and physical care, technological tasks, and management of symptoms, pain, and medication. ${ }^{11-13,21,28-30}$ Furthermore, it encompasses the psychosocial aspects of care, including the social, psychological, and spiritual needs of the patient. Competent training ensures caregivers have access to healthcare providers, services, and/or equipment that are coordinated, competent, organized, flexible, adequate, communicative, continuous, and consistent.

Mentorship is central to empowerment and sustainability in the caregiving role, given that caregivers are ambivalent about their own needs to be met. ${ }^{7,31-34}$ At a minimum, mentorship is needed to address the following critical areas: navigating the healthcare system; caregiver health and wellness (including self-care and minimizing social isolation); family dynamics; communication and assertiveness skills; sharing the care; and bereavement. Caregivers who are also involved in paid employment may also need to be mentored in making use of caregiver-friendly workplace policies, such as flexible work or unpaid leave. As with the education and training needs already outlined, there is no one best communication medium or program to ensure this mentorship, but rather needs to be tailored to each caregiver and caregiving situation.

Given these dimensions, caregiver education, training, and mentorship ideally take place in person, by a palliative navigator or, if unavailable, trained individuals associated with one of a number of existing programs and services available in geographically proximate community-based organizations, such as the Family Caregivers Network of British Columbia, or disease-specific organizations, such as the Canadian Cancer Society or the Alzheimer's Society of Canada. These individuals ideally are healthcare professionals trained in palliative care. They should be competent in providing faceto-face education, training, and mentorship, as well as being able to direct caregivers to other educational resources such as on-line platforms, videos, and hard-copy formats.

\section{Conclusions}

The Ispos Reid survey results provide a starting point from which caregiver preferences are revealed. The rapid review of literature, which is placed in context through real-world research experience, sets forth a set of findings and results 
specific to the resources required to ensure adequate education, training, and mentorship for caregivers of Canadians experiencing a life-limiting illness. After first introducing the burden of care, the preferences for caregiver education, and the importance of the patient-caregiver dyad, the palliative navigator approach is presented as a key role in the education, training, and mentorship of caregivers.

\section{Acknowledgments}

The author acknowledges the contributions of the Palliative Care Matters Research Support Team: Sharna Polard and Roger Salus for library services and literature searching; Heather Stiles and Michelle Sims for overall research coordination and assistance; and Lisa Weisgerber for literature screening, literature review, and project support.

\section{Author Disclosure Statement}

No competing financial interests exist.

\section{References}

1. Holm M, Årestedt K, Carlander I, et al.: Short-term and long-term effects of a psycho-educational group intervention for family caregivers in palliative home care? Results from a randomized control trial. Psychooncology 2016;25: 795-802.

2. Martín-Carrasco M, Domínguez-Panchón AI, GonzálezFraile E, et al.: Effectiveness of a psychoeducational intervention group program in the reduction of the burden experienced by caregivers of patients with dementia: The EDUCA-II randomized trial. Alzheimer Dis Assoc Disord 2014;28:9-87.

3. Morris LC: What to expect when a death is expected: Development of an educational program for home hospice caregivers: Support for the final days of a life. A Caregiver Guide [Master's thesis]. California: University of California, Davis, 2013.

4. Ventura AD, Burney S, Brooker J, et al.: Home-based palliative care: A systematic literature review of the selfreported unmet needs of patients and carers. J Palliat Med 2014;28:391-402.

5. Williams AM, Wang L, Kitchen P: Differential impacts of care-giving across three caregiver groups in Canada: Endof-life care, long-term care and short-term care. Health Soc Care Community 2014;22:187-196.

6. Collinge W, Kahn J, Walton T, et al.: Touch, caring, and cancer: Randomized controlled trial of a multimedia caregiver education program. Support Care Cancer 2013;21: 1405-1414.

7. IPSOS-Reid. IPSOS 2016: Reference Document \& Financial Report. https://www.ipsos.com/sites/default/files/ 201704/Ipsos_2016_Reference_document_VA.pdf. 2017 (accessed June 16, 2017).

8. Duggleby W, Penz K, Goodridge D, et al.: The transition experience of rural older persons with advanced cancer and their families: A grounded theory study. BMC Palliat Care 2010;9:1-9.

9. Grande G, Stajduhar K, Aoun S, et al.: Supporting lay carers in end of life care: Current gaps and future priorities. J Palliat Med 2009;23:339-344.

10. Ewing G, Grande G: Development of a Carer Support Needs Assessment Tool (CSNAT) for end-of-life care practice at home: A qualitative study. J Palliat Med 2012; 27:244-256.

11. Stajduhar K, Funk L, Toye C, et al.: Part 1: Home-based family caregiving at the end of life: A comprehensive review of published quantitative research (1998-2008). Palliat Med 2010;24:573-593.

12. Stajduhar K, Funk L, Jakobsson E, Ohlen J: A critical analysis of health promotion and 'empowerment' in the context of palliative family care-giving. Nurs Inq 2010;17: 221-230.

13. Stajduhar KI, Funk L, Outcalt L: Family caregiver learning-How family caregivers learn to provide care at the end of life: A qualitative secondary analysis of four datasets. J Palliat Med 2013;27:657-664.

14. Greene A, Aranda S, Tieman JJ, et al.: Can assessing caregiver needs and activating community networks improve caregiver-defined outcomes? A single-blind, quasiexperimental pilot study: Community facilitator pilot. J Palliat Med 2012;26:917-923.

15. Duggleby W, Robinson CA, Kaasalainen S, et al.: Developing navigation competencies to care for older rural adults with advanced illness. Can J Aging/La Rev Can du Vieil 2016;35:206-214.

16. Williams AM, Donovan R, Stajduhar K, Spitzer D: Cultural influences on palliative family caregiving: Service recommendations specific to the Vietnamese in Canada. BMC Res Notes 2015;8:1.

17. Donovan R, Williams A: Shifting the burden: The effects of home-based palliative care on family caregivers in rural areas. Health in Rural Canada 2011;6:316.

18. Novik N, MacLean M: Pain and palliative care with seniors in Canada's Northern Territories. In: Kulig JC, Williams AM (eds): Health in Rural Canada. Toronto: UBC Press, 2011, pp. 334.

19. Williams AM, Eby JA, Crooks VA, et al.: Canada's compassionate care benefit: Is it an adequate public health response to addressing the issue of caregiver burden in endof-life care? BMC Public Health 2011;11:1.

20. DeMiglio L, Williams AM: A qualitative study examining the sustainability of shared care in the delivery of palliative care services in the community. BMC Palliat Care 2013; 29:12:1.

21. Funk LM, Stajduhar KI, Outcalt L: What family caregivers learn when providing care at the end of life: A qualitative secondary analysis of multiple datasets. Palliat Support Care 2015;13:425-433.

22. Street AF, Swift K, Annells M, et al.: Developing a webbased information resource for palliative care: An actionresearch inspired approach. BMC Med Inform Decis Mak 2007;7:26.

23. Kazuki S, Mitsunori M, Tatsuya M, et al.: The long-term effect of a population-based educational intervention focusing on end-of-life home care, life-prolongation treatment, and knowledge about palliative care. J Palliat Care 2009;25:206-212.

24. Arcand M, Brazil K, Nakanishi M, et al.: Educating families about end-of-life care in advanced dementia: Acceptability of a Canadian family booklet to nurses from Canada, France, and Japan. Int J Palliat Nurs 2013;19:6774.

25. Bekelman DB, Nowels CT, Retrum JH, et al.: Giving voice to patients' and family caregivers' needs in chronic heart failure: Implications for palliative care programs. J Palliat Med 2011;14:1317-1324. 
26. Dikkers MF, Dunning T, Savage S: Information needs of family carers of people with diabetes at the end of life: A literature review. J Palliat Med 2013;16:16171623.

27. Docherty A, Owens A, Asadi-Lari M, et al.: Knowledge and information needs of informal caregivers in palliative care: A qualitative systematic review. J Palliat Med 2008; 22:153-171.

28. Holm M, Carlander I, Fürst C, et al.: Delivering and participating in a psycho-educational intervention for family caregivers during palliative home care: A qualitative study from the perspectives of health professionals and family caregivers. BMC Palliat Care 2015;14:1-10.

29. Klemm PR, Hayes ER, Diefenbeck CA, Milcarek B: Online support for employed informal caregivers: Psychosocial outcomes. Comput Inform Nurs 2014;32:10-20.

30. Schildmann EK, Higginson IJ: Evaluating psychoeducational interventions for informal carers of patients receiving cancer care or palliative care: Strengths and limitations of different study designs. Palliat Med 2011;25: 345-356.

31. Harrop E, Byrne A, Nelson A: "It's alright to ask for help": Findings from a qualitative study exploring the in- formation and support needs of family carers at the end of life. BMC Palliat Care 2014;13:1-10.

32. Angelo JK, Egan R, Reid K: Essential knowledge for family caregivers: A qualitative study. Int J Palliat Nurs 2013;19:383-388.

33. Leow M, Chan S, Chan M: A pilot randomized, controlled trial of the effectiveness of a psychoeducational intervention on family caregivers of patients with advanced cancer. Oncol Nurs Forum 2015;42:E63-E72.

34. Thomas K, Moore G: The development and evaluation of a multimedia resource for family carers of patients receiving palliative care: A consumer-led project. Palliat Support Care 2013;13:417-423.

Address correspondence to: Allison M. Williams, PhD Gender, Work, and Health School of Geography and Earth Sciences McMaster University 1280 Main Street West Hamilton, Ontario L8S 4K1

Canada

E-mail: awill@mcmaster.ca 